\title{
Soft Actuators and Robots that Are Resistant to Mechanical Damage
}

\section{Citation}

Martinez, Ramses V., Ana C. Glavan, Christoph Keplinger, Alexis I. Oyetibo, and George M. Whitesides. 2014. "Soft Actuators and Robots That Are Resistant to Mechanical Damage." Advanced Functional Materials 24 (20) (February 1): 3003-3010. doi:10.1002/adfm.201303676.

\section{Published Version}

doi:10.1002/adfm.201303676

\section{Permanent link}

http://nrs.harvard.edu/urn-3:HUL.InstRepos:25753288

\section{Terms of Use}

This article was downloaded from Harvard University's DASH repository, and is made available under the terms and conditions applicable to Open Access Policy Articles, as set forth at http:// nrs.harvard.edu/urn-3:HUL.InstRepos:dash.current.terms-of-use\#OAP

\section{Share Your Story}

The Harvard community has made this article openly available.

Please share how this access benefits you. Submit a story.

Accessibility 


\title{
Submitted to
}

DOI: 10.1002/adfm. 2013XXXXX.

\section{Soft Actuators and Robots that Are Resistant to Mechanical Damage}

By Ramses V. Martinez $z^{1 \ddagger}$, Ana C. Glavan ${ }^{1 \ddagger}$, Christoph Keplinger ${ }^{1}$, Alexis I. Oyetibo ${ }^{1}$, and George M. Whitesides ${ }^{1,2,3^{*}}$

[*] Prof. G. M. Whitesides

${ }^{1}$ Department of Chemistry and Chemical Biology, Harvard University, 12 Oxford Street, Cambridge, MA 02138, (USA).

${ }^{2}$ Kavli Institute for Bionano Science \& Technology, Harvard University, 29 Oxford Street, Cambridge, MA 02138, (USA).

${ }^{3}$ Wyss Institute for Biologically Inspired Engineering, Harvard University, 60 Oxford Street, Cambridge, MA 02138, (USA).

E-mail: gwhitesides@gmwgroup.harvard.edu

Dr. R. V. Martinez, A. C. Glavan, Dr. C. Keplinger, Alexis I. Oyetibo

${ }^{1}$ Department of Chemistry and Chemical Biology, Harvard University, 12 Oxford Street, Cambridge, MA 02138, USA.

Keywords: Soft Robotics, Impact-Resistance, Pneumatic Actuators, Elastomers, Composites, Soft Machines.

\begin{abstract}
This paper characterizes the ability of soft pneumatic actuators and robots to resist mechanical insults that would irreversibly damage or destroy hard robotic systems—-systems fabricated in metals and structural polymers, and actuated mechanically_of comparable sizes. The pneumatic networks that actuate these soft machines are formed by bonding two layers of elastomeric or polymeric materials that have different moduli on application of strain by pneumatic inflation; this difference in strain between an extensible top layer and an inextensible, strain-limiting, bottom layer causes the pneumatic network to expand anisotropically. While all the soft machines described here are, to some extent, more resistant to damage by compressive forces, blunt impacts, and severe bending than most corresponding hard systems, the composition of the strain-limiting layers confers on them very different tensile and compressive strengths.
\end{abstract}




\section{Introduction}

Robots based on rigid structural elements—metallic skeletons, electrical motors, conventional mechanical actuators, and mechanical joints — can be superb at performing tasks that require high precision, rapid movement, or application of high levels of force or power. These "hard" robots are, however, often heavy, costly, and difficult (and expensive) to control. ${ }^{[1-3]}$ Hard robots designed to be lightweight and capable of performing delicate operations are vulnerable to damage by impact or by compression; joints and sensors are especially vulnerable to bending, collisions, and blunt impacts, since even small deformations can lead to incorrect positioning of their components and to failure. ${ }^{[4,5]}$

Robots that resist damage by pressure or impact would be useful in operations that expose them to damaging mechanical insults-e.g., by falling, impact, or other crushing forces. Examples of relevant applications of inexpensive, light, damage-resistant actuators and machines include search and rescue, and the exploration of human-unfriendly, dangerous, unstable sites.

“Soft” machines_robots fabricated largely using elastomeric structural elements— offer an alternative to conventional "hard" machines. ${ }^{[6-12]}$ Soft actuators, for example, take advantage of the anisotropic expansion under pressure of a network of elastomeric pneumatic chambers connected by small channels to accomplish, with relative simplicity, types of complex motions and tasks for which conventional hard machines would require complex control systems and large numbers of components. ${ }^{[6-10]}$ Moreover, because they are fabricated from elastomers, soft actuators can resist certain kinds of damage (such as blunt impacts or severe bending) better than hard robotic structures (and also most animals of comparable weight and size). We wished to quantify the range of physical damage that soft machines, 
fabricated largely in silicone elastomers, can withstand without suffering changes in mechanical performance.

This paper characterizes the resistance to physical damage of soft pneumatic actuators based on composite structures comprising a highly elastomeric siloxane (Ecoflex) and a strain-limiting layer or core of either PDMS or a composite flexible material (such as paper or Nylon mesh embedded in an elastomeric matrix). We tested the resistance of these actuators to uniaxial forces, compressive loads, severe bending, transient pressures (impacts) and blunt impacts.

\section{Background}

Many different strategies to improve the impact resistance of hard robots have been met with various degrees of success. ${ }^{[13,14]}$ The German Aerospace Center (DLR) has developed and commercialized torque-controlled lightweight robots (LWRs) comprising arms or hands. ${ }^{[13,15]}$ These actuators were built using lightweight, damage-resistant materials such as urethane resins. The compliance of these systems, particularly of their joints, remains an issue. ${ }^{[5]}$ The vulnerability of the joints can be diminished by introducing mechanical compliance into their design, or by dynamically decoupling the rotational inertia of the actuator from the joints and connectors when an impact occurs; these changes necessarily bring complexity and expense. Spring-based transmissions have been used as compliant

joints; their use, however, decreases performance as a consequence of the oscillations and settling time with which they are associated. ${ }^{[5]}$

A different strategy to improve impact resistance in hard robots is to introduce mechanically compliant components in the robot to shield the rest of the structure from 
impacts. "Sand Flea”, for example, is a 5-kg robot that drives like a remote-controlled car on flat terrain, but, using a cylindrical launcher powered by pressurized carbon dioxide, can jump up to nine meters into the air to overcome obstacles. ${ }^{[16]}$ This robot relies on its four oversized plastic wheels, with diameters larger than the thickness of its body, to absorb the impact forces on landing. ${ }^{[17]}$ Similarly, other jumping robots such as "Jollbot” or "Sandia hopper" use an external shock-absorbing shell to damp shock impulse, and dissipate kinetic energy of a landing after vertical leaps of up to four meters. ${ }^{[18,19]}$ Greater impacts, however, will saturate the shock absorbers on these robots, and cause damage when their hard components absorb parts of the kinetic energies of the impacts.

Shape-memory Alloys (SMAs)—alloys that “remember” their original, cold-forged shape and that return to their pre-deformed shape on heating-have been used to make worm-like soft robots. ${ }^{[11,20,21]}$ For example, “GoQBot”- a caterpillar-inspired rolling robot— has a soft elastomeric body with two sections, each of which houses a tensile actuator that provides bending for inching movements. ${ }^{[11]}$ The soft body of this robot protected it from crashes with speeds over $0.8 \mathrm{~m} / \mathrm{s}^{[11]}$ "Meshworm” is an earthworm-like robot fabricated from nickel titanium (NiTi) SMA coiled springs. This robot stretches and contracts to crawl across the ground using a sequential antagonistic motion achieved using NiTi coil actuators. This soft robot can withstand certain mechanical insults, exemplified by the crushing pressures encountered when "meshworm” is stepped on by a human. ${ }^{[21]}$

We have previously demonstrated the flexibility of pneumatic networks (pneu-nets) embedded in elastomeric structures as actuators for soft structures. ${ }^{[6-10]}$ These structures are, in essence, only slabs of elastomeric polymers (sometimes including embedded, flexible, sheets, meshes, or fibers) surrounding channels filled with gas; we expect them to be 
significantly more resistant to blunt impacts, stretching, and bending than hard robots of similar size and weight. Here we test that expectation.

\section{Results and Discussion}

\subsection{Choice of Materials}

We chose embedded pneumatic networks (pneu-nets) to supply energy to our soft machines because the low viscosity of air enables rapid actuation. ${ }^{[9]}$ Air is also lightweight and widely available. The degrees of freedom of the actuation are determined by the size and distribution of the pneumatic channels, which expand upon pressurization. We have described

the design of the soft robotic structures previously (starfish, ${ }^{[6]}$ quadrupeds, ${ }^{[7]}$ and tentacles ${ }^{[8]}$ ).

We fabricated multi-channel structures molded in a flexible silicone elastomer

(Ecoflex 00-30, Smooth-on, http://www.smooth-on.com) using standard soft lithographic

techniques. ${ }^{[22]}$ A flat membrane with typical thickness between 0.5 and $2 \mathrm{~mm}$ was used to seal the channels in the pneumatic network, and to serve as a strain-limiting layer. This strain-limiting layer was fabricated using a silicone elastomer (polydimethylsiloxane (PDMS, Dow Corning Sylgard 184)), or a composite of polyester/cellulose blend paper (VWR, West Chester, PA), or a Nylon mesh (Small Parts, Inc.) embedded in Ecoflex. These materials are easily accessible, relatively inexpensive, non-toxic, and easily shaped. Moreover, they can be bonded to each other to form multilayer and 3D structures. We acquired force data using an Instron 5544A electromechanical testing machine at $1000 \mathrm{~Hz}$. 


\subsection{Characterization of the Resistance of Soft Actuators and Robots to Tension and}

\section{Compression}

In previous work, we described the fabrication and performance of different classes of soft pneumatic machines such as starfish-shaped grippers, ${ }^{[6]}$ quadrupeds, ${ }^{[7]}$ and tentacles. ${ }^{[8]}$ Figure 1 illustrates these devices and their characteristics. We used these actuators (with different embedded strain-limiting layers) to study the resistance of this type of soft devices to several common sources of mechanical damage. We tested the mechanical performance of the most vulnerable parts of the soft actuators-legs of the soft starfish and quadrupeds, 5-cm

long pieces of soft tentacle. ${ }^{[6-8]}$ After each test, the structures were examined for local failures (i.e., tears or "aneurysms"-ruptures of the internal walls of the pneu-net) and function (“ability to grip” for starfish and tentacles, “locomotion” for quadrupeds).

We subjected the soft machines to uniaxial forces in order to characterize the deformability of their elastomeric components. These soft actuators elongate in the direction of the force to accommodate the deformation until the maximum strain before failure is reached. We characterized the maximum strain before failure of the soft actuators using the strain at break coefficient $\left(\varepsilon_{b}\right)$ defined by Eq. $1 .{ }^{[23]}$ Here, $l_{0}$ is the length of the soft machine when no external tensile or compressive stress is applied and $l_{b}$ is the length of the device at the point of failure. The parameter $\varepsilon b$ is dimensionless, and takes positive values for tensile strain, and negative values for compressive strain.

$$
\varepsilon_{b}=\frac{l_{b}-l_{0}}{l_{0}}
$$

The tensile strength (TS) is the maximum tensile stress to which a material can be subjected before failure. ${ }^{[23]}$ The compressive strength (CS) is the maximum compressive 
stress to which a material can be subjected before failure. Intuitively, both TS and CS measure the mechanical stress required to bring a material to the point of failure, while $\varepsilon b$ measures the deformation that a material can withstand before failure occurs.

\subsection{Resistance to Tensile Strain}

Figure 2 shows that soft robots fabricated entirely from elastomers (Ecoflex and PDMS, in our case) exhibit maximum strain at break of $\varepsilon b \sim 1.2$, and provides evidence that these soft robots can accommodate higher axial extensions than small animals with hard skeletons (such as rodents or small birds) ${ }^{[24]}$ or hard robots of comparable weight and sizes. ${ }^{[25]}$ The cortical bone in vertebrates has a $\varepsilon_{b} \sim 0.012,{ }^{[26]}$ while the tentacular stalk of a squid can tolerate strains as high as $0.8^{[27]}$ (although strains higher than 0.2 cause irreversible damage to the giant nerve fiber embedded in the stalk). ${ }^{[28]}$ The values of $\varepsilon b$ for entirely elastomeric soft robots are consistent with experimental values of the elongation at break of pure PDMS; ${ }^{[29]}$ the tensile strength of these robots is, however, relatively low (TS $\sim 150 \mathrm{kPa}$ for soft robotic tentacles, and $\sim 400 \mathrm{kPa}$ for soft robotic quadrupeds and starfish grippers), comparable to the tensile strength of the squid mantle $(\mathrm{TS}=310 \mathrm{kPa}) .{ }^{[30]}$ To increase the resistance of the soft robots to tensile forces, we introduced different materials in the strain limiting layers of these robots. These flexible strain-limiting layers have a much higher stiffness than pure elastomers and allow the soft robot to withstand higher loads while still maintaining its "soft", flexible characteristics. Fig. 2a shows that a soft tentacle with a strain-limiting core comprising an embedded bungee cord can withstand an axial extension stress of $\sim 8.25 \mathrm{MPa}$. Soft starfish grippers and quadrupeds with embedded nylon mesh (Fig. 2b,c) tolerate axial extension stress up to 2.50 MPa without breaking. These values exceed the typical values of tensile force per unit area that would damage hard robotic systems (and especially their vulnerable components, such as joints) of comparable size. ${ }^{[5]}$ 
Toughness is the ability of a material to absorb energy and deform without fracturing. The values of the toughness of the soft actuators will indicate, therefore, the maximum amount of energy that they can absorb without failing. Toughness can be determined by measuring the area underneath the stress-strain curve, as defined by Eq. ${ }^{[23]}$ Here $T$ is the toughness $\left(\mathrm{J} / \mathrm{m}^{3}\right), \varepsilon$ is the strain (dimensionless), and $\sigma$ is the stress $(\mathrm{Pa})$ :

$$
T=\int_{0}^{\varepsilon_{b r e a k}} \sigma d \varepsilon
$$

An embedded bungee cord provided soft robotic tentacles the highest toughness of the series $\left(710 \mathrm{~kJ} / \mathrm{m}^{3}\right.$, Figure 3c); a strain-limiting core made of rope provided the lowest corresponding toughness. Among starfish grippers and quadrupeds, the highest toughness $\left(\sim 400 \mathrm{~kJ} / \mathrm{m}^{3}\right)$ is observed for stain-limiting layers with embedded nylon mesh, and the lowest for stain-limiting layers made of PDMS.

\subsection{Resistance to Compressive Strain}

Soft robots based on elastomers can also tolerate a wide range of compressive forces due to the isotropic elastic properties of their elastomeric components-materials sometimes used as shock-absorbents. ${ }^{[29]}$ As elastomers are incompressible "quasi-fluids", they expand along directions perpendicular to the compressive force to accommodate the deformation. Figure 4 shows that actuators that use PDMS in strain-limiting layers or cores fail under compressive stress higher than 2.6 MPa, when PDMS cracks, having exceeded its maximum deformation at break. ${ }^{[29]}$ When using materials with higher stiffness (such as a paper sheet or Nylon mesh) embedded in strain-limiting layers, the failure of the soft machines occurs when the lateral expansion induced by the compressive force causes the delamination of the pneumatic layer from the embedded material. For composite strain-limiting layers, the failures 
occur at compressive stresses between $9.60 \mathrm{MPa}$ for paper and 10.2 MPa for Nylon mesh (as non-extensible layers). Animals of sizes and weights comparable to these soft machines are significantly more vulnerable than these soft machines to compression damage. For example, the maximum compressive stress tolerated by mouse vertebrae is $\sim 2.5 \mathrm{MPa} .^{[31]}$ Compressive stresses above $200 \mathrm{kPa}$ break the axoplasm of the giant nerve fiber embedded in the stalk of a squid, and irreversibly damage it. ${ }^{[32]}$

Figure 5 compares the tolerance to compressive force of a soft robotic tentacle and a stainless steel cylinder with similar dimensions $(\mathrm{OD}=12 \mathrm{~mm}$, wall thickness $=1 \mathrm{~mm}$, length $=2 \mathrm{~cm}$ ) and comparable weights $(2.4 \mathrm{~g}$ for the soft tentacle fragment, and $5.6 \mathrm{~g}$ for the stainless steel cylinder). The stainless steel cylinder—a representative component of "hard" robots fabricated using metals-is irreversibly damaged when a compressive force of $\sim 1800 \mathrm{~N}$ is applied. When the same compressive force is applied to a soft tentacle, its elastic body deforms without material failure, and allows the system to recover its original shape when the load is removed.

\subsection{Resistance to Transient Pressure (Impacts)}

The elastomeric matrix of soft machines can efficiently absorb and distribute the force of impacts over their entire structure. ${ }^{[33]}$ This characteristic allows soft robots based on elastomers to tolerate a wide range of blunt impacts; examples include being dropped on concrete, hammered vigorously or even run over by a car.

Soft tentacles resist being thrown onto flat concrete surfaces with velocities of at least $27 \mathrm{~m} / \mathrm{s}$ (the highest value we measured quantitatively) without changing their response to pneumatic pressure (see Figure S8). We predict that these soft machines will survive falls 
from any height (within the atmosphere) if we estimate their terminal velocity $\left(\mathrm{V}_{\mathrm{T}}\right)$ to be $\mathrm{V}_{\mathrm{T}} \sim 12.7 \mathrm{~m} / \mathrm{s}$ (see Supporting Information); at this velocity, the soft actuator will experience transient pressures of $\sim 30 \mathrm{kPa}$ during a 5 ms impact with the concrete (see Movie_M5). Our soft actuators can resist compressive stresses one order of magnitude higher (in the order of MPa, see Fig. 3).

Figure 6 shows snapshots of a quadruped being repeatedly struck with a steel hammer. Videos of the experiment, Movie_M1 and Movie_M2, are included in the Supporting Information. We found that the robot kept crawling without any apparent changes to its gait after multiple strikes from the hammer.

We tested the resistance of these soft machines quantitatively to high transient pressures by subjecting them to substantial mechanical insults. Figure 7a shows snapshots of a car (a 2012 Ford Focus Hatchback), running over a soft robotic gripper using paper as strain-limiting layer (see Movie_M3). Despite the compressive force exerted by a $1450 \mathrm{~kg}$ motor vehicle, the soft gripper did not show any visible signs of damage or changes in its ability to grasp and hold objects. The nylon mesh strain-limiting layer also provided some resistance to puncture hazards such as broken glass. Figure $7 \mathrm{~b}$ shows snapshots of the same car running over a soft robotic gripper placed on top of a pile of fragments of crushed glass (see Movie_M4).

\subsection{Resistance to Severe Bending, Twisting, and Wadding}

Figure 8 shows that soft actuators based on flexible elastomers are undamaged by bending with a small radius of curvature. This ability of soft robots to resist bending in any part of their structure is an important advantage of soft robots over hard robots of the same 
size. Fig. 8a-c shows a soft robotic tentacle being twisted and tied in three overhand knots. Upon untying, the function of the tentacle is restored ( $\mathrm{N}=7$ actuators). A starfish is bent at 180-degree angle (Fig. 8d-f). Upon release of a compression load of $1.2 \mathrm{kN}$, the starfish is actuated and experiences no loss of function ( $\mathrm{N}=7$ actuators). A quadruped is wadded with two consecutive 180-degree bends and compressed $(1.2 \mathrm{kN})$, but after release it returns to a normal gait ( $\mathrm{N}=7$ actuators).

\section{Conclusions}

This paper describes the resistance of soft actuators such as grippers, quadrupeds, and tentacles to mechanical damage by axial extension, or due to compressive forces, transient impacts, and bending that would damage animals or "hard" robotic systems of similar weight and size. These robots are built entirely from commonly available materials, such as paper, rope, or nylon mesh, and elastomers, yet displays remarkable resistance to a wide range of mechanical insults. When more highly developed, these soft robotic structures have the potential to be useful as soft machines that can operate in congested and hazardous spaces. Several characteristics make them especially well-suited for these applications: i) they can be fabricated simply, rapidly and sufficiently inexpensively that they can be considered for single-use applications; ii) they can be light (with a low loading in their contact with ground); iii) they are simple to actuate and control; iv) they are undamaged after being severely bent, dropped, hammered, folded, and wadded. Before this study, we might have assumed that to build tougher soft robots (i.e. able to absorb large amounts of energy without mechanical damage) would require new, sophisticated materials and mechanical designs. In fact, common materials may provide enough toughness (at least to compressive damage) for many immediate applications of these devices. 
The soft machines described here, at the present level of development, also have two limitations: i) Soft robots based on pneu-nets are typically less resistant than hard robots to penetrating trauma, sharp punctures, and high temperatures. We have recently described the use of alternative composite materials that minimize the damage to the pneumatic channels of grippers when manipulating sharp objects; ${ }^{[34]}$ ii) They are not presently capable of exerting the same level of force as a hard robot with equivalent mass. Circumventing this limitation will require the use of different materials and designs.

\section{Experimental}

Fabrication of Micropneumatic Soft Machines. We used computer-aided-design (CAD) software (Alibre, Inc.) to design a negative mold of the pneumatic network of the soft actuators. We fabricated the negative mold in acrylonitrile butadiene styrene (ABS) using a three-dimensional (3D) printer (StrataSys Dimension Elite, Eden Prairie, MN). We cast the pneumatic network of the actuators using a two-part silicone rubber (Ecoflex 00-30, SmoothOn Inc., Easton PA.) Figure S1 and Figure S2 describe the molds used to fabricate quadrupeds and starfish grippers and the design of the respective pneumatic networks.

Figure S3 illustrates the design of the molds we used to fabricate soft tentacles, according to the method described before. ${ }^{[8]}$ Details of the procedure used to fabricate the strain-limiting layers and to bond them to the pneumatic layers, as well as the procedure used to connect the soft robot to an off-board pressure source, are described in the Supporting Information.

Force-deformation Measurements. The force-deformation curves were recorded using an electromechanical test frame (Model 5544A, Instron, Norwood MA) equipped with a load cell with a capacity of either $1000 \mathrm{~N}$ or $10000 \mathrm{~N}$, operated at a crosshead speed of $5 \mathrm{~mm} / \mathrm{min}$. Typically, seven test specimens were used for each material in each test method. To securely 
grip the specimen and reduce the potential for damage (tearing, puncturing) caused by gripping, we placed four layers of paper/polyester fabric between the metallic grips of the testing machine and the specimen (see Figure S4 for details). Methods recorded load (N) vs. axial extension (mm) or load $(\mathrm{N})$ vs. axial compression (mm) curves, at a data acquisition rate of 10000 points per minute. Figure S6 shows the parameters used to describe the stress-strain curve of a soft actuator.

\section{Acknowledgements}

DARPA supported the design and fabrication phases of this work under contracts W911NF11-1-0094 and W911NF-09-1-0476. The testing of the non-linear mechanical properties of the soft actuators under stress was supported by the Department of Energy (DE-FG0200ER45852). R.V.M. acknowledges funding by the FP7 People program under the project Marie Curie IOF-275148, as well as the support of the Madrid Institute for Advanced Studies, IMDEA Nanoscience, Calle Faraday 9, Ciudad Universitaria de Cantoblanco, 28049 Madrid, Spain. A.I.O. acknowledges funding by the NSF Biomaterials Research Initiative Dedicated to Gateway Experiences (BRIDGE) REU program at Harvard (NSF DMR-1262895). We thank Dr. Rui M. D. Nunes for his help during the mechanical testing of the actuators. Supporting Information is available online from Wiley InterScience or from the website of the Whitesides group (http://gmwgroup.harvard.edu/pubs)

¥ These authors contributed equally to this work.

* Corresponding authors email: gwhitesides@gmwgroup.harvard.edu

Received: ((will be filled in by the editorial staff))

Revised: ((will be filled in by the editorial staff)) Published online: ((will be filled in by the editorial staff)) 


\section{References}

[1] M. Richard, R. Clavel, Mech. Sci. 2011, 2, 99.

[2] Y. Yun, Y. Li, Nonlinear Dynam. 2010, 61, 829.

[3] B. Chen, Z. C. Pei, Z. Y. Tang, X. Q. Guo, H. X. Zhong, Adv. Sci. Lett. 2012, 8, 433.

[4] N. O. Pérez-Arancibia, K. Y. Ma, K. C. Galloway, J. D. Greenberg, R. J. Wood, Bioinspir. Biomim. 2011, 6, 036009.

[5] A. Albu-Schaffer, O. Eiberger, M. Grebenstein, S. Haddadin, C. Ott, T. Wimbock, S. Wolf, G. Hirzinger, IEEE. Robot. Autom. Mag. 2008, 15, 20.

[6] F. Ilievski, A. D. Mazzeo, R. F. Shepherd, X. Chen, G. M. Whitesides, Angew. Chem., Int. Ed. 2011, 123, 1930.

[7] R. F. Shepherd, F. Ilievski, W. Choi, S. A. Morin, A. A. Stokes, A. D. Mazzeo, X.

Chen, M. Wang, G. M. Whitesides, Proc. Natl. Acad. Sci. U. S. A. 2011, 108, 20400.

[8] R. V. Martinez, J. L. Branch, C. R. Fish, L. Jin, R. F. Shepherd, R. Nunes, Z. Suo, G. M. Whitesides, Adv. Mater. 2012.

[9] R. V. Martinez, C. R. Fish, X. Chen, G. M. Whitesides, Adv. Funct. Mater. 2012, 22, 1376.

[10] S. A. Morin, R. F. Shepherd, S. W. Kwok, A. A. Stokes, A. Nemiroski, G. M. Whitesides, Science 2012, 337, 828.

[11] H. T. Lin, G. G. Leisk, B. Trimmer, Bioinspir. Biomim. 2011, 6, 026007.

[12] E. Brown, N. Rodenberg, J. Amend, A. Mozeika, E. Steltz, M. R. Zakin, H. Lipson, H. M. Jaeger, Proc. Natl. Acad. Sci. U. S. A. 2010, 107, 18809.

[13] H. Liu, P. Meusel, G. Hirzinger, M. Jin, Y. Liu, Z. Xie, IEEE-ASME T. Mech. 2008, 13, 461.

[14] Q. M. Li, Q. H. Qin, S. W. Zhang, H. Deng, Appl. Mech. Mat. 2011, 44, 743. 
[15] U. Hagn, M. Nickl, S. Jörg, G. Passig, T. Bahls, A. Nothhelfer, F. Hacker, L. Le-Tien, A. Albu-Schäffer, R. Konietschke, Ind. Robot. 2008, 35, 324.

[16] E. Ackerman, IEEE Spectrum Robot. Blog 2012.

[17] Boston Dynamics, SandFlea Jumping Robot, retrieved at http://www.bostondynamics.com/img/SandFlea Datasheet v1_0.pdf 2012.

[18] M. Kovač, M. Schlegel, J. C. Zufferey, D. Floreano, Auton. Robot. 2010, 28, 295.

[19] P. Weiss, Sci. News 2001, 159, 88.

[20] Y. K. Wang, C. N. Song, Z. L. Wang, C. Guo, Q. Y. Tan, in Intelligent Computing and Information Science, Vol. 135 (Ed: R. Chen), Springer Berlin Heidelberg, 2011, 619. [21] S. Kim, E. Hawkes, K. Choy, M. Joldaz, J. Foleyz, R. Wood, "Micro artificial muscle fiber using NiTi spring for soft robotics", presented at IEEE/RSJ International Conference on Intelligent Robots and Systems IROS, 2009.

[22] Y. Xia, G. M. Whitesides, Ann. Rev. Mat. Sci. 1998, 28, 153.

[23] W. F. Smith, J. Hashemi, Foundations of Materials Science and Engineering, McGraw-Hill Publishing, New York 2006.

[24] A. A. Biewener, J. Exp. Biol. 1982, 98, 289.

[25] "Robot Materials", in http://www.robotbooks.com/robot-materials.htm, 2013.

[26] T. M. Keaveny, E. F. Morgan, G. L. Niebur, O. C. Yeh, Annu. Rev. Biomed. Eng. 2001, 3, 307.

[27] W. M. Kier, J. L. VanLeeuwen, J. Exp. Biol. 1997, 200, 41.

[28] J. A. Galbraith, D. R. Matteson, L. E. Thibault, J. Biomech. Eng. 1993, 115, 13.

[29] F. Schneider, T. Fellner, J. Wilde, U. Wallrabe, J. Micromech. Microeng. 2008, 18, 065008.

[30] J. D. Kuo, H. O. Hultin, M. T. Atallah, B. S. Pan, J. Agric. Food Chem. 1991, 39, 1149. 
[31] M. F. Vaz, H. Canhão, J. E. Fonseca, in Advances in Composite Materials - Analysis of Natural and Man-Made Materials, (Ed: D. P. Tesinova), Available from: http://www.intechopen.com/books/advances-in-composite-materials-analysis-of-natural-andman-made-materials/bone-a-composite-natural-material, InTech 2011.

[32] P. Gallant, J. Neuropathol. Exp. Neurol. 1992, 51, 220.

[33] T. Sugaiwa, H. Iwata, S. Sugano, "Shock absorbing skin design for human-symbiotic robot at the worst case collision", presented at 8th IEEE-RAS International Conference on Humanoid Robots, 2008.

[34] R. F. Shepherd, A. A. Stokes, R. M. D. Nunes, G. M. Whitesides, Adv. Mater. 2013. 
Fig. 1

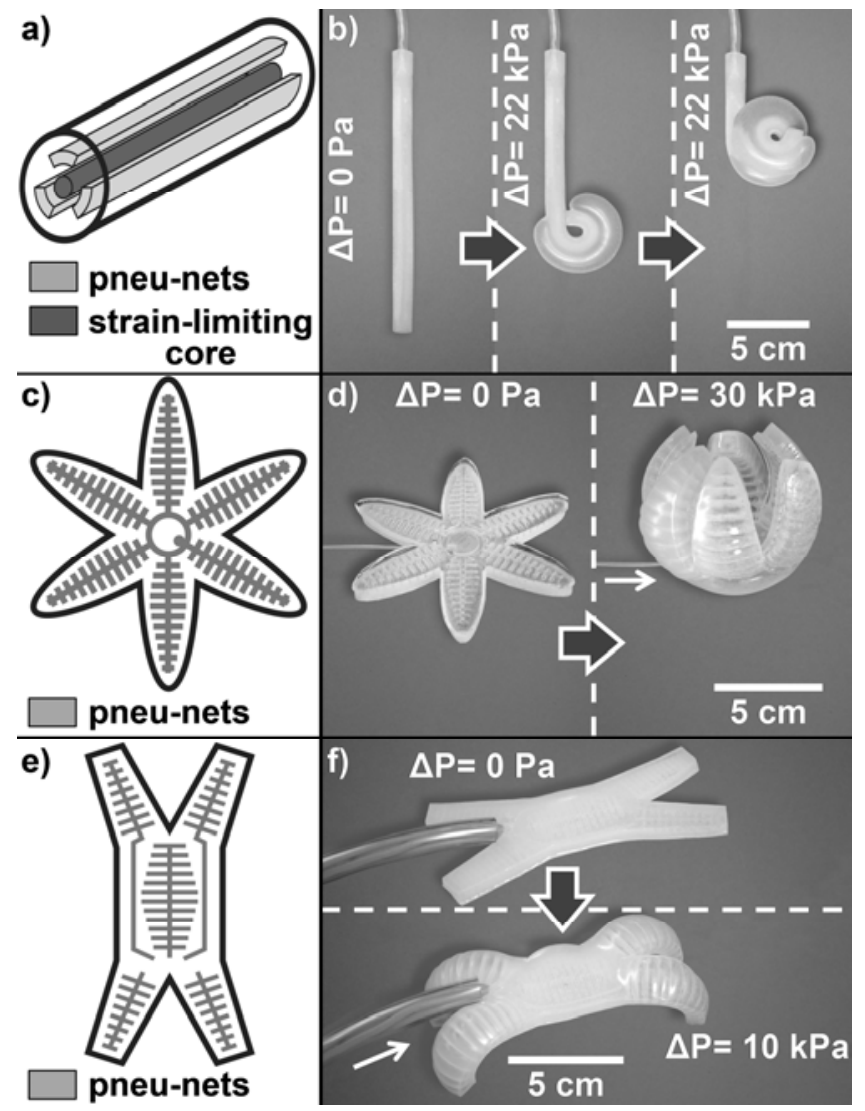

Figure 1. Soft actuators used to test the mechanical damage resistance of soft robots.

a) Schematic design of the pneu-nets of the tentacle. b) Actuation of a tentacle with threedimensional mobility. c) Design and d) actuation of a starfish-shaped gripper. e) Design and f) actuation of a quadruped capable of walking using several gaits. 


\section{Fig. 2}
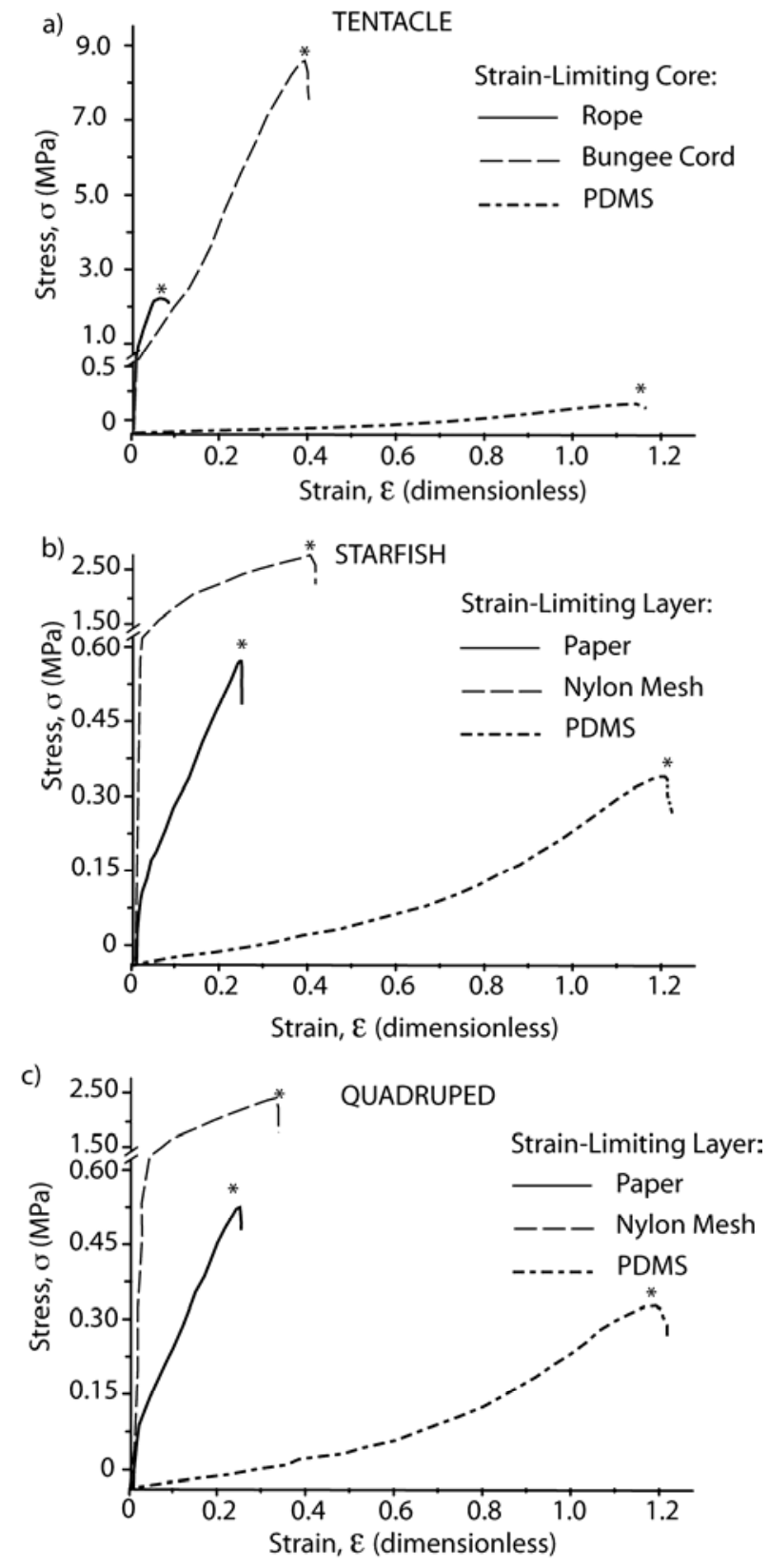

Figure 2. Typical stress-strain curves for soft machines with different embedded materials and composites. a) Soft tentacles with PDMS, rope, or bungee cord as strain-limiting cores. b) Starfish gripper and c) quadrupeds, with PDMS, paper, or nylon mesh as strain-limiting layers. The points of failure are marked in the graph by asterisks. 


\section{Fig. 3}
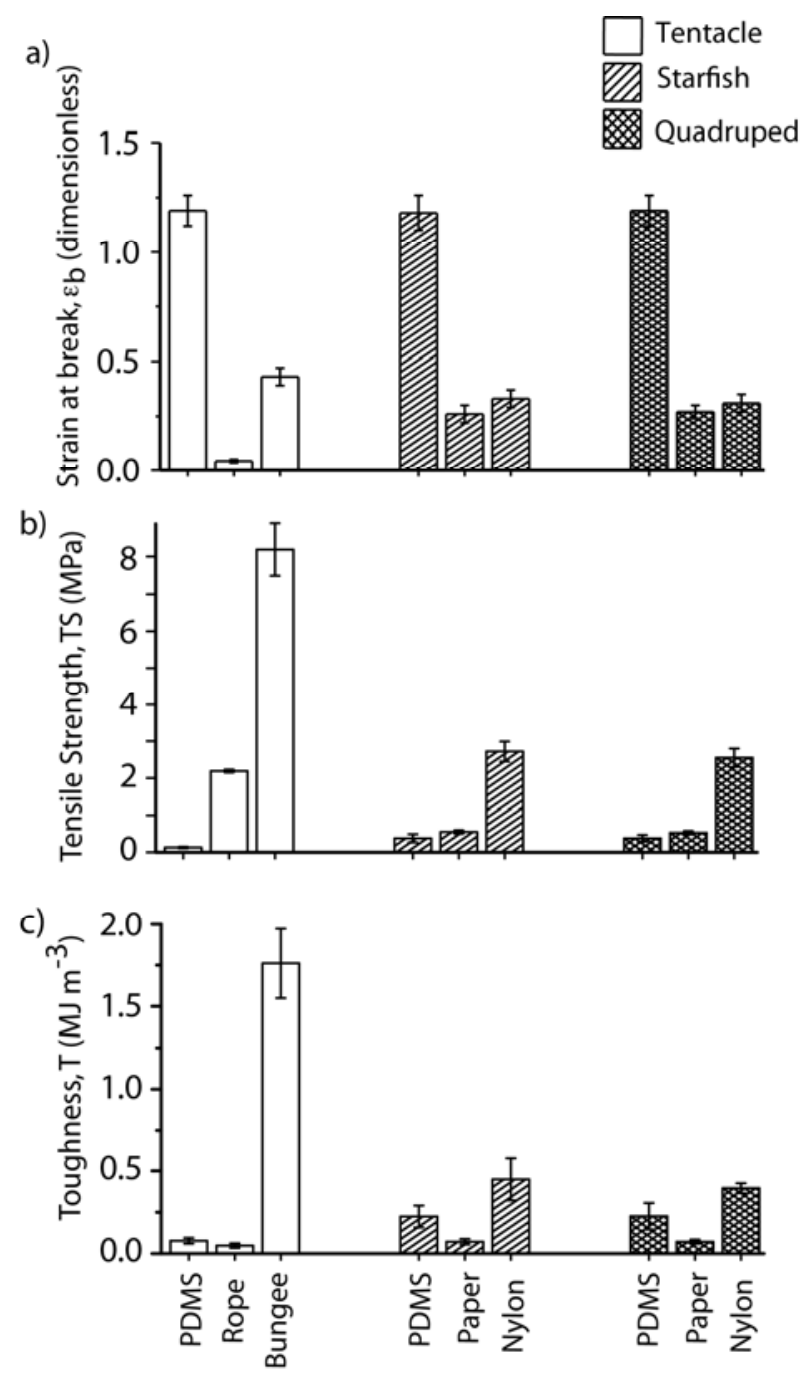

Figure 3. Values of the strain at break (a), the tensile strength (b), and toughness (c) of soft machines as a function of the composition of their respective strain limiting layers. 
Fig. 4

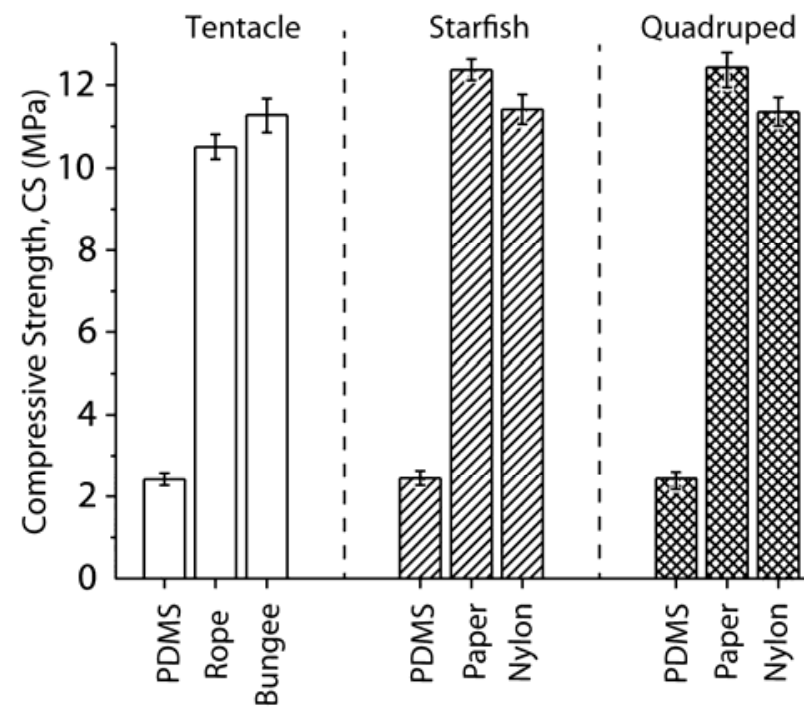

Figure 4. Values of compressive strength for different actuators as a function of the composition of their respective strain limiting layers. 
Fig. 5

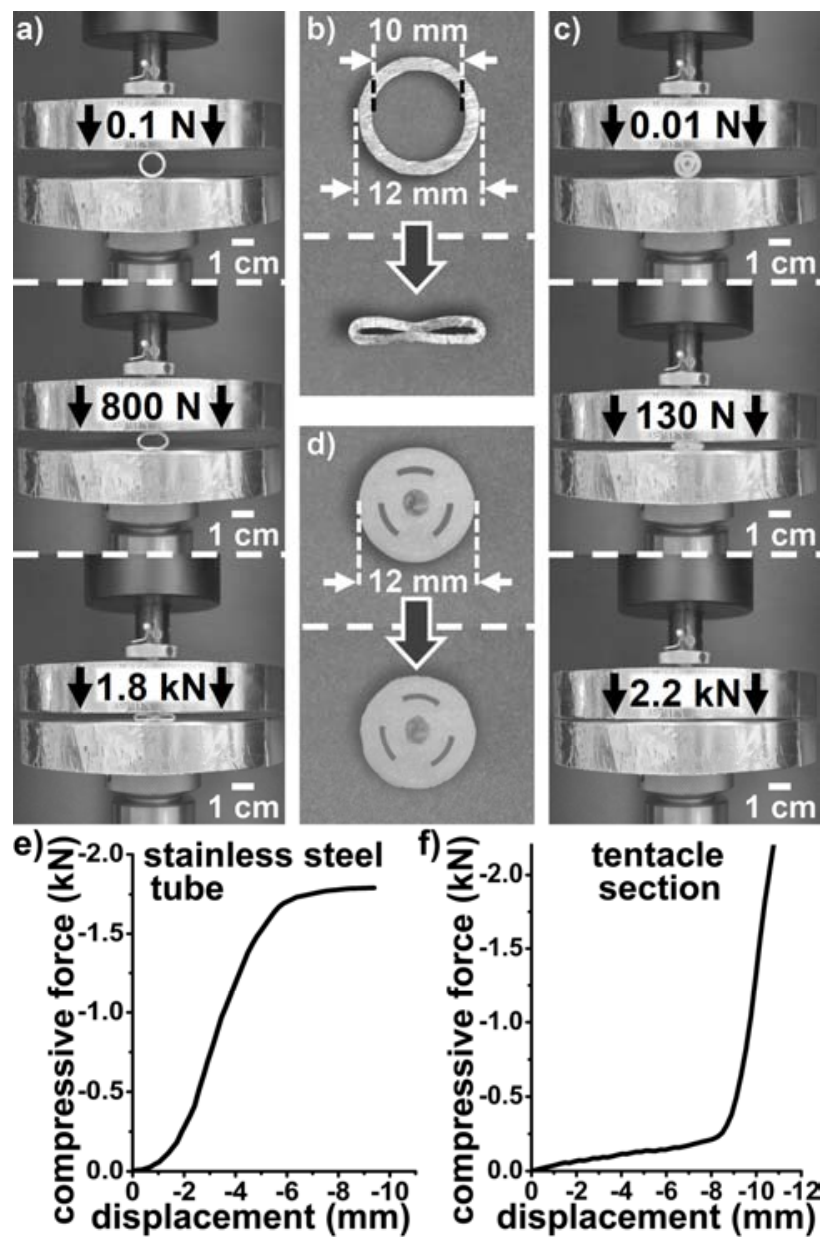

Figure 5. Comparison between resistances to compressive forces for a soft-robotic tentacle and a stainless steel cylinder, with the same diameter and length. a) Compressive test of a stainless steel cylinder. b) Images of the metallic tube before and after being subjected to a compressive load of $1.8 \mathrm{kN}$, corresponding to a compressive stress of $7.5 \mathrm{MPa}$.

c) Compressive test of a soft tentacle with a bungee cord as strain-limiting core. d) Images of the soft tentacle before and after being subjected to a compressive load of $2.2 \mathrm{kN}$, corresponding to a compressive stress of $~ 9.2 \mathrm{MPa}$. e), f) Compressive strain as a function of compressive load applied for the stainless steel tube and the soft tentacle. 


\section{Fig. 6}

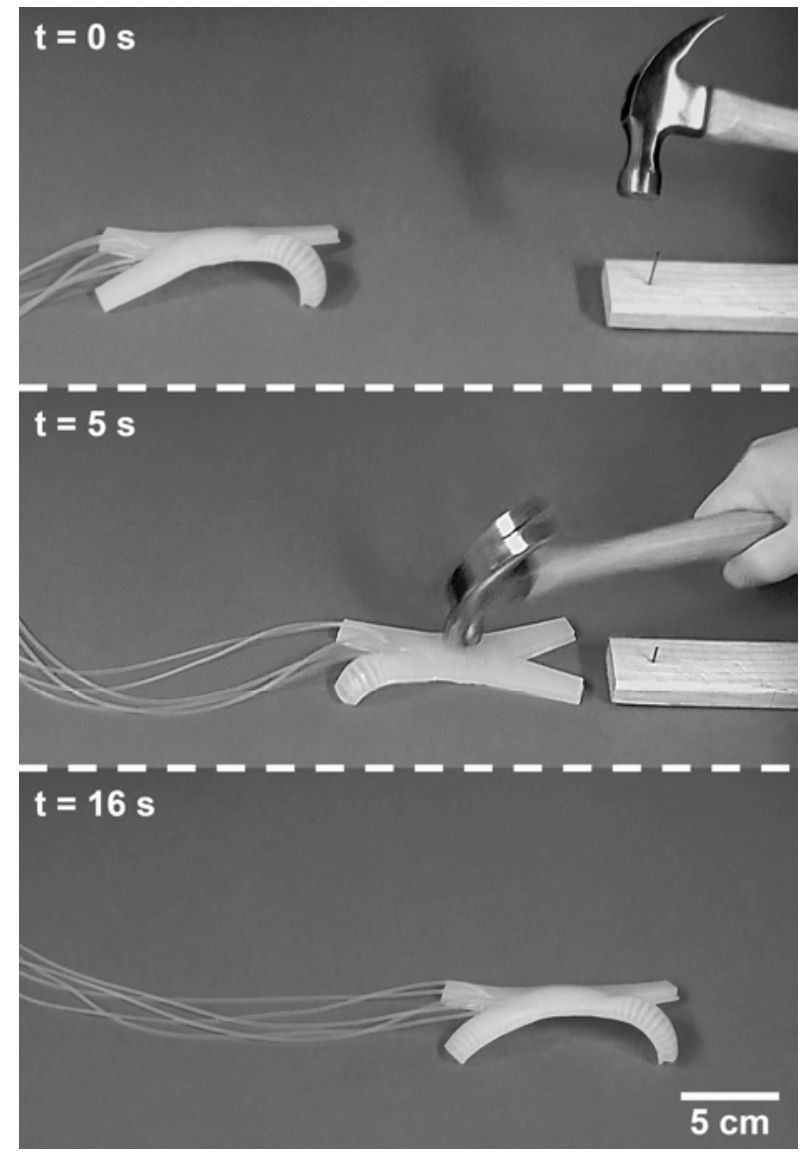

Figure 6. Snapshots of a quadruped with a Nylon mesh strain-limiting layer being repeatedly struck with a hammer while walking. The power of the hammer strike is sufficient to drive a nail into a piece of wood. The tubes that supply gas to the pneumatic network are depicted on the left. See Supporting Information, Movie_M2 for the full movie. 
Fig. 7

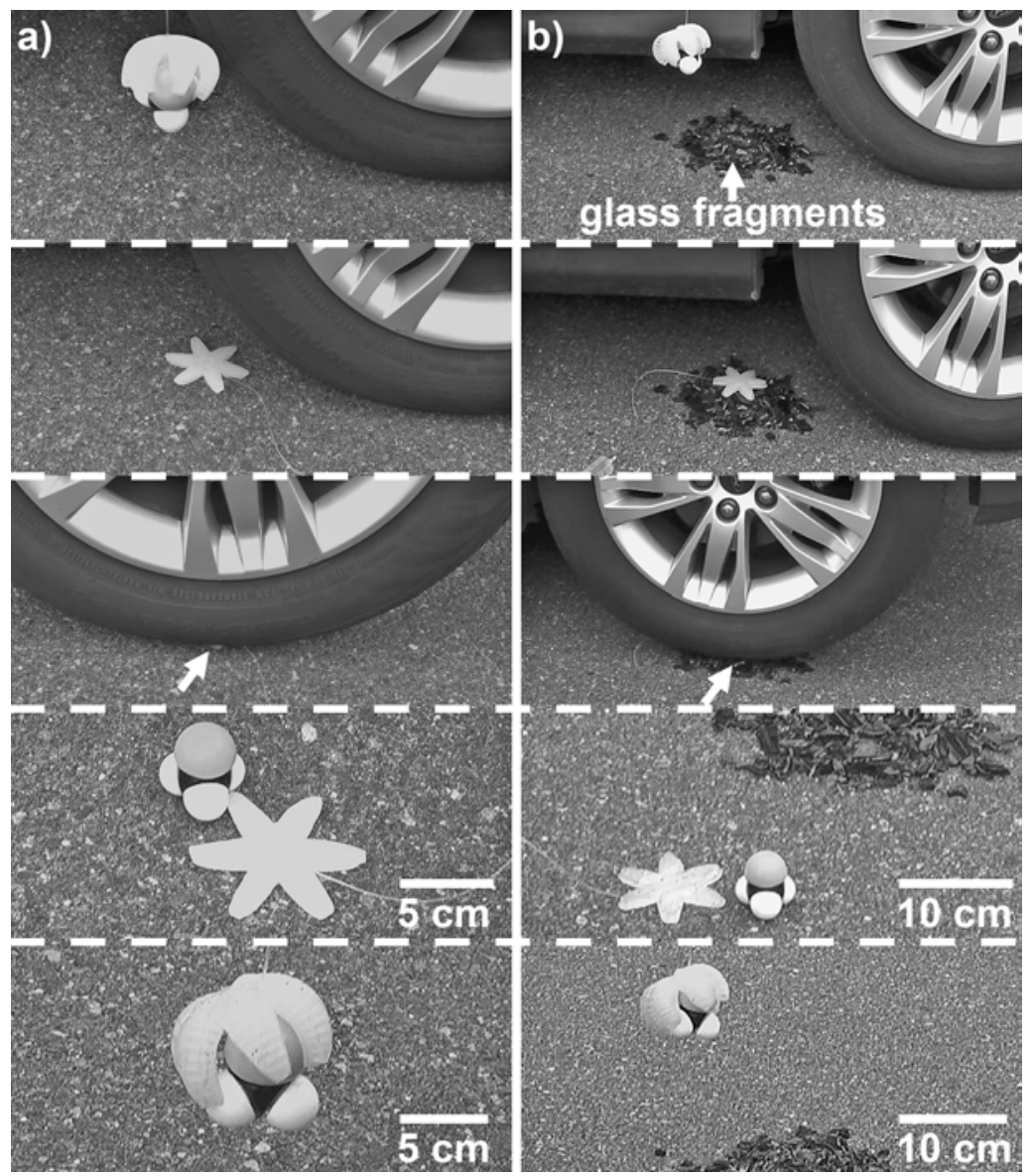

Figure 7. a) Snapshots of an elastomeric gripper with a paper strain-limiting layer being run over by a $1450 \mathrm{~kg}$ Ford Focus Hatchback. The vehicle exerts a compressive force of $\sim 3.6 \mathrm{kN}$, or compressive stress of up to $1.71 \mathrm{MPa}$ over the surface of the starfish. b) Snapshots of an elastomeric gripper with a Nylon mesh strain-limiting layer being run over by the same car when resting over a pile of pieces of crushed glass. The Nylon mesh embedded in the strain-limiting layer protected the pneumatic layer from puncturing. In both cases the gripper is still functional after being run over by the vehicle. See Supporting Information, Movie_M3 and Movie_M4 for the full movies. 
Fig. 8
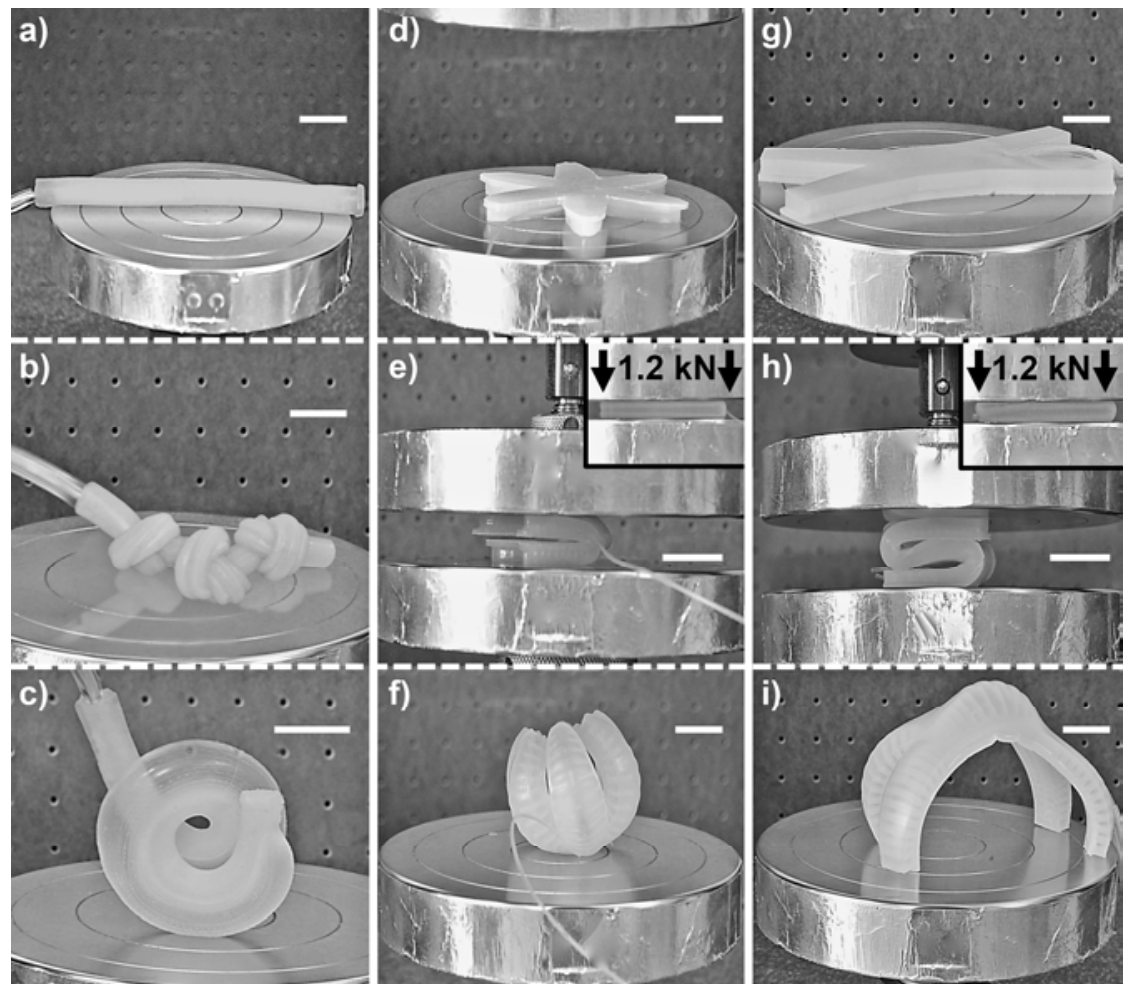

Figure 8. a) A soft robotic tentacle is b) twisted, stretched, and tied in three overhand knots.

c) The function of the tentacle is restored upon untying, ( $N=7$ actuators). d) A starfish is

e) bent at 180-degree angle and compressed under a load of $1.2 \mathrm{kN}$. f) The starfish is released and actuated without experiencing loss of function ( $\mathrm{N}=7$ actuators). g) A quadruped is

h) wadded with two consecutive 180-degree bends and compressed under a load of $1.2 \mathrm{kN}$.

i) The quadruped returns to a normal gate after being released ( $\mathrm{N}=7$ actuators). 


\section{Table of Contents}

Soft pneumatic actuators and robots based on composites consisting of elastomers and embedded flexible reinforcing sheets are significantly more resistant to blunt impacts, tensile forces, and severe bending than hard robotic systems soft similar size and weight. We quantify the range of physical damage that soft robots and actuators can withstand without suffering changes in mechanical performance.

Keywords: Soft Robotics, Impact-Resistant, Pneumatic Actuators, Elastomers, Composites, Soft Machines.

Ramses V. Martinez ${ }^{\ddagger}$, Ana C. Glavan ${ }^{\ddagger}$, Christoph Keplinger, Alexis I. Oyetibo, and George M. Whitesides*

Soft Actuators and Robots that Are Resistant to Mechanical Damage.

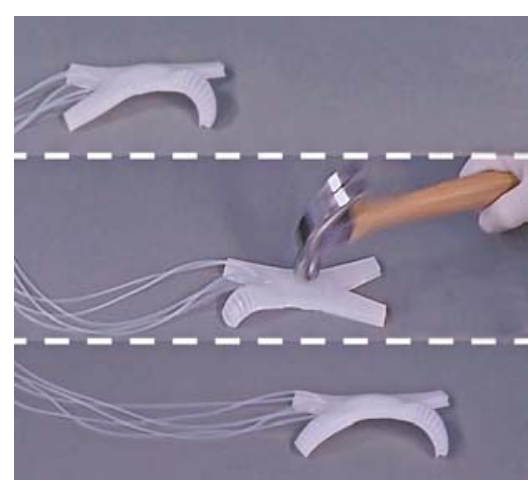

